\title{
A relação entre ansiedade e o tratamento odontológico: pesquisa de campo
}

\author{
The relationship between anxiety and dental treatment: field research \\ La relación entre ansiedad y tratamiento dental: tema de investigación
}

Recebido: 16/10/2021 | Revisado: 24/10/2021 | Aceito: 25/10/2021 | Publicado: 29/10/2021

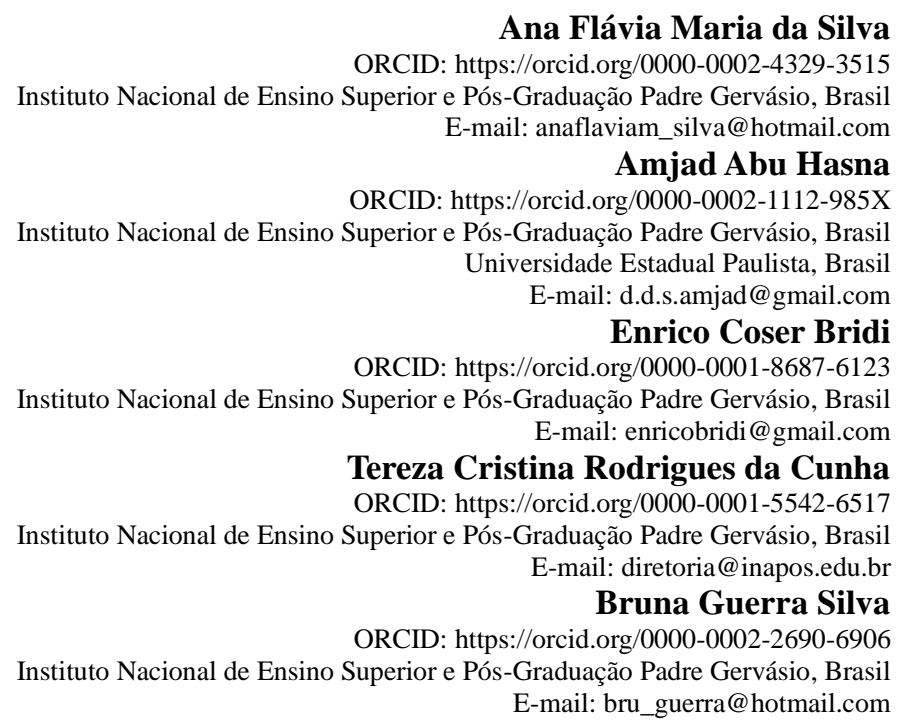

\section{Resumo}

Objetivo: Este trabalho foi realizado para identificar os maiores fatores responsáveis pelo desencadeamento de ansiedade nos pacientes atendidos em clínica escola de odontologia. Metodologia: total de 87 participantes (entre 1870 anos) foram incluidos neste estudo após autorização dos mesmos por meio de um Termo de Consentimento Livre e Esclarecido. Foi utilizado um questionário para avaliar a ansiedade frente ao tratamento odontológico. Este trabalho foi realizado na disciplina de clínica integrada do Instituto de Ensino Superior e Pós-Graduação Padre Gervásio INAPÓS após aprovação do comitê de ética em pesquisa que envolve seres humanos da Universidade Estadual de Campinas (no 4.989.109). Os dados obtidos foram apresentados em porcentagem. Resultados: Dos 87 voluntários, $28,7 \%$ relataram ter medo de dentista, sendo que a agulha $(22,4 \%)$ e o medo de sentir dor $(32,7 \%)$ foram os principais fatores apontados como responsáveis pelo desencadeamento da ansiedade durante a consulta odontológica; 27,5\% já vivenciaram alguma experiência negativa no consultório odontológico e 14\% já deixaram de se consultar, mesmo em casos de urgência, por medo. Foi perguntado aos participantes da pesquisa, dentre algumas opções, quais lhe proporcionariam um atendimento mais tranquilo e os mais citados foram: conhecer o procedimento antes do mesmo ser realizado $(61,7 \%)$ e ouvir música durante a consulta $(27,6 \%)$. Sobre a espera pelo atendimento e a forma como são abordados na chegada ao consultório $34,4 \%$ e $49,4 \%$ dos pacientes relataram um aumento na ansiedade, respectivamente. Em relação a postura do cirurgião dentista, 28,7\% julgaram ter muita influência na ansiedade frente ao tratamento odontológico e sobre os EPI'S utilizados pelos cirurgiões dentistas, 50,5\% dos entrevistados se sentem confortáveis e seguros com a utilização dos mesmos. Conclusão: Mediante a análise dos resultados obtidos, pode-se observar que o medo de dentista existe e os fatores responsáveis por desencadearem essa insegurança começam desde o momento do agendamento da consulta.

Palavras-chave: Medo; Ansiedade; Tratamento odontológico.

\begin{abstract}
Objective: This study aimed to identify the main factors responsible for triggering anxiety in patients undergoing dental care at a dental school. Methodology: a total of 87participants (aged 18-70 years) were included in this study after their authorization through an Informed Consent Form. A questionnaire was used to assess anxiety regarding dental treatment. This work was carried out in the integrated clinic of the National Institute of Higher Education and Post-graduation Priest Gervásio "INAPÓS" after approval by the Ethics Committee for Research involving Human Beings at the University of Campinas (N. 4,989,109). The data obtained were presented in percentage. Results: Of the 87 volunteers, $28.7 \%$ reported being afraid of the dentist, and the needle $(22.4 \%)$ and fear of feeling pain $(32.7 \%)$ were the main factors identified as responsible for triggering anxiety during the dental appointment; $27.5 \%$ have
\end{abstract}


already experienced some negative experience in the dental office and $14 \%$ have interrupted the treatment, even in urgent cases, out of fear. The survey participants were asked, among some options, which would provide them with a calmer service and the most mentioned were: knowing the procedure before it is performed (61.7\%) and listening to music during the consultation $(27.6 \%)$. About waiting for care and how they are approached upon arrival at the office, $34.4 \%$ and $49.4 \%$ of patients reported an increase in anxiety, respectively. Regarding the posture of the dental surgeon, $28.7 \%$ thought it had a great influence on anxiety regarding dental treatment and on the PPE used by dental surgeons, $50.5 \%$ of respondents felt comfortable and safe with their use. Conclusion: Through the analysis of the results obtained, it can be observed that the fear of the dentist exists and the factors responsible for triggering this insecurity start from the moment of scheduling the appointment.

Keywords: Fear; Anxiety; Dental treatment.

\section{Resumen}

Objetivo: Este estudio tuvo como objetivo identificar los principales factores responsables de desencadenar ansiedad en pacientes sometidos a cuidados odontológicos en una facultad de odontología. Metodología: se incluyó en este estudio a un total de 87 participantes (de 18 a 70 años) después de su autorización a través de un Formulario de Consentimiento Informado. Se utilizó un cuestionario para evaluar la ansiedad ante el tratamiento odontológico. Este trabajo se llevó a cabo en la Clínica Integrada del Instituto Nacional de Educación Superior y Posgrado Sacerdote Gervásio "INAPÓS" luego de la aprobación del Comité de Ética para la Investigación con Seres Humanos de la Universidad de Campinas (No. 4,989,109). Los datos obtenidos se presentaron en porcentaje. Resultados: De los 87 voluntarios, el $28,7 \%$ refirió tener miedo al dentista, siendo la aguja $(22,4 \%)$ y el miedo a sentir dolor $(32,7 \%)$ los principales factores identificados como responsables de desencadenar ansiedad durante la consulta odontológica; El $27,5 \%$ ya ha vivido alguna experiencia negativa en el consultorio odontológico y el $14 \%$ ha interrumpido el tratamiento, incluso en casos urgentes, por miedo. A los encuestados se les preguntó, entre algunas opciones, cuáles les brindaría un servicio más tranquilo y las más mencionadas fueron: conocer el procedimiento antes de realizarlo $(61,7 \%)$ y escuchar música durante la consulta (27,6\%). Sobre la espera de atención y cómo se les aborda a su llegada a la consulta, el $34,4 \%$ y el $49,4 \%$ de los pacientes informaron un aumento de la ansiedad, respectivamente. En cuanto a la postura del cirujano dentista, el $28,7 \%$ opinó que tenía una gran influencia en la ansiedad con respecto al tratamiento odontológico y en el EPI utilizado por los cirujanos dentistas, el 50,5\% de los encuestados se sintió cómodo y seguro con su uso. Conclusión: A través del análisis de los resultados obtenidos, se puede observar que existe el miedo al dentista y los factores responsables de desencadenar esta inseguridad comienzan desde el momento de programar la cita.

Palabras clave: Medo; Ansiedade; Tratamento odontológico.

\section{Introdução}

A ansiedade é um sentimento vago e desagradável de medo, apreensão, caracterizado por tensão ou desconforto derivado de antecipação de perigo, de algo desconhecido ou estranho (Castillo, Recondo, Asbahr, \& Manfro, 2000; do Nascimento, da Silva Araújo, Gusmão, \& Cimões, 2011; Heyman et al., 2016). Para muitos pacientes o atendimento odontológico é sinônimo de estresse e ansiedade (Lin et al., 2021). De acordo com (Kanegane, Penha, Borsatti, \& Rocha, 2003), aproximadamente um quarto da população americana adulta evitou fazer visitas regulares ao dentista devido às experiências negativas vivenciadas durante a infância.

Muitos são os fatores apontados como gatilho para o desencadeamento de ansiedade frente ao tratamento odontológico, sendo a dor é apontada como um desses fatores estressantes (Pohjola, Rekola, Kunttu, \& Virtanen, 2016) além dos fatores psicológicos (De Stefano, 2019), de acordo com (Possobon, Carrascoza, Moraes, \& Costa Jr, 2007), pacientes ansiosos tendem a superestimar a dor que sentiram e a recordar-se das experiências desagradáveis com mais intensidade do que provavelmente ocorreram, ainda mais, o primeiro contato com o cirurgião dentista, geralmente durante a infância, ditará como será sua relação com a odontologia pelo resto da vida. Além disso, a ansiedade é relacionada com o alfabetismo em saúde bucal, que diz respeito a capacidade de obter e processar a informação que lhes foi dada sobre à odontologia (Barasuol, Busato, Felipak, \& Menezes, 2016).

O medo é associado a uma saúde bucal deficiente, essa associação é feita porque pacientes ansiosos tendem a procurar atendimento odontológico somente em casos de urgência, muitas vezes necessitando de tratamentos mais invasivos (Appukuttan, 2016; Doganer et al., 2017; Murad, Ingle, \& Assery, 2020). Estes tratamentos exacerbam e reforçam seu medo 
fazendo com que haja esquiva de tratamento futuro novamente. Desta maneira, instala-se um ciclo vicioso quando o paciente não é tratado de forma adequada. Estar bem preparado para identificar com eficiência e oferecer um tratamento adequado baseado nos receios individuais de cada paciente é de extrema importância para a condução de um atendimento seguro sem maiores desgastes para ambos os envolvidos. O principal objetivo do cirurgião dentista deve ser acolher o paciente e aliviar seus medos e ansiedade para que eles se sintam motivados a longo prazo para futuras consultas odontológicas (Appukuttan, 2016; Fux-Noy et al., 2019; Randall, Shulman, Crout, \& McNeil, 2014).

Sendo assim, o objetivo deste trabalho foi identificar os maiores fatores responsáveis pelo desencadeamento de ansiedade nos pacientes atendidos em clínica escola durante o atendimento odontológico. A hipótese nula foi que o medo do paciente não se afeta pelo medo do próprio dentista.

\section{Metodologia}

Foi realizado um estudo quantitativo através de um questionário destinados aos pacientes atendido na Clínica Escola do Instituto de Ensino Superior e Pós-Graduação Padre Gervásio - INAPÓS. No total foram abordados 87 voluntários, de ambos os sexos compreendidos entre 18 a 70 anos. Enquanto os pacientes aguardavam pelo atendimento foram convidados a participar da pesquisa, aqueles que estavam de acordo assinaram o Termo de Consentimento Livre e Esclarecido autorizando a sua participação e responderam a um questionário, contendo 10 perguntas objetivas de múltipla escolha, onde foram avaliados os seguintes itens: medo de dentista, a origem do medo, a influência da postura do cirurgião-dentista no medo desencadeado pelo paciente e alternativas para diminuição da ansiedade durante o atendimento. Este estudo foi realizado após aprovação do comitê de ética em pesquisa que envolve seres humanos da Universidade Estadual de Campinas (No. 4,989,109).

Critérios de inclusão: pacientes da Clínica Escola do Instituto de Ensino Superior e Pós-Graduação Padre Gervásio INAPÓS, Pouso Alegre, MG, pacientes adultos maiores de 18 anos, pacientes que responderam os questionários por completo

Critérios de exclusão: indivíduos que não sejam pacientes da Clínica Escola - INAPÓS, pacientes menores de 18 anos, pacientes que não responderam os questionários por completo.

O questionário foi composto de 9 questões:

Questão 1. Você tem medo de dentista? Sim / Não

Questão 2. Se a sua resposta foi sim a pergunta anterior, qual (is) é a origem do seu medo? A agulha da anestesia/ O anestésico/ As canetas de alta e baixa rotação (motorzinho)/ O consultório odontológico/ A vestimenta do cirurgião-dentista/ Instrumentais pontiagudos/ Dor/ Outros. Qual (is):

Questão 3. Já vivenciou alguma experiência negativa em uma consulta odontológica? Sim / Não (Quais: - trismo após procedimento de exodontia/ dor durante o procedimento/ trauma durante a infância/ extração sem anestesia/ muita dor na anestesia em um procedimento da infância/ muita dor na colocação de pino/graduanda desmaiou durante a realização de exodontia/ falta de diálogo com o cirurgião dentista/ a anestesia não pegou/ ortodontista degastou muito meu dente/ abcesso fênix após o tratamento de canal/ insatisfação com o resultado do procedimento/ extração e restauração errada/ tinha só um buraquinho e o dentista acabou com o meu dente/ dentista agressivo.

Questão 4. Já deixou de se consultar, mesmo em casos de urgência, por medo? Sim / Não

Questão 5. Qual (is) dos elementos abaixo te proporcionariam um atendimento mais tranquilo? Música durante o atendimento/ Cirurgião-dentista usando uma cor diferente do branco/ Ser atendido assistindo seu programa favorito de televisão/ Conhecer o procedimento antes do mesmo ser realizado/ Outro.

Questão 6. O tempo de espera até o atendimento, aumenta a sua ansiedade? Sim / Não

Questão 7. Quando você agenda uma consulta, o tempo de espera até a data marcada altera a sua qualidade de vida? Sim / Não 
Questão 8. Quanto a postura do cirurgião dentista, desde o seu primeiro contato com ele até o momento da realização do procedimento, influencia no seu medo? muito/ pouco/ mediano/ não tem influência.

Questão 9. Os equipamentos de proteção individuais utilizados pelos cirurgiões dentistas, principalmente durante o período de pandemia, despertam em você algum sentimento? causam pouco desconforto/ causam muito desconforto/ me confortam/ não causam nada.

Os dados obtidos foram apresentados em porcentagem.

\section{Resultados e Discussão}

Na questão de número 1 foi perguntado aos pacientes se eles tinham medo de dentista. Dos 87 voluntários, 28,7\% responderam de forma afirmativa (Figura.1)

Figura1. Distribuição de respostas da questão de número 1.

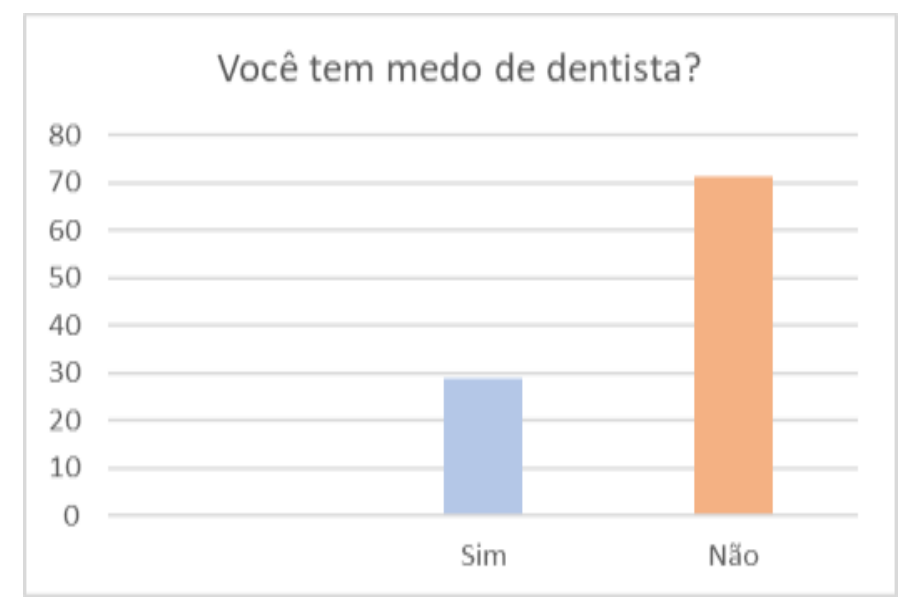

Fonte: Autores.

A pergunta seguinte listava alguns objetos que poderiam estar relacionados a origem do medo desencadeado durante a consulta odontológica, e dentre as opções as mais citadas foram: a agulha (24,7\%) e o medo de sentir dor (32,7\%) (Figura.2).

Figura 2. Distribuição de respostas da questão de número 2.

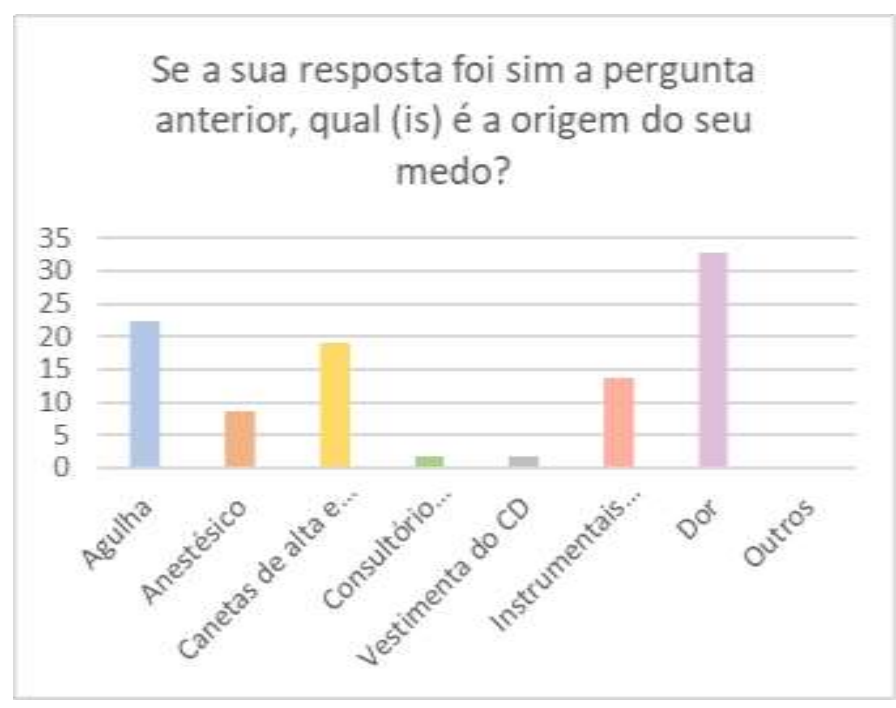




\section{Fonte: Autores.}

Total de 24 dos entrevistados relataram já ter vivenciado alguma experiência negativa no consultório odontológico, o que corresponde a 27,5\% dos participantes (Figura 3).

Figura 3. Distribuição de respostas da questão de número 3.

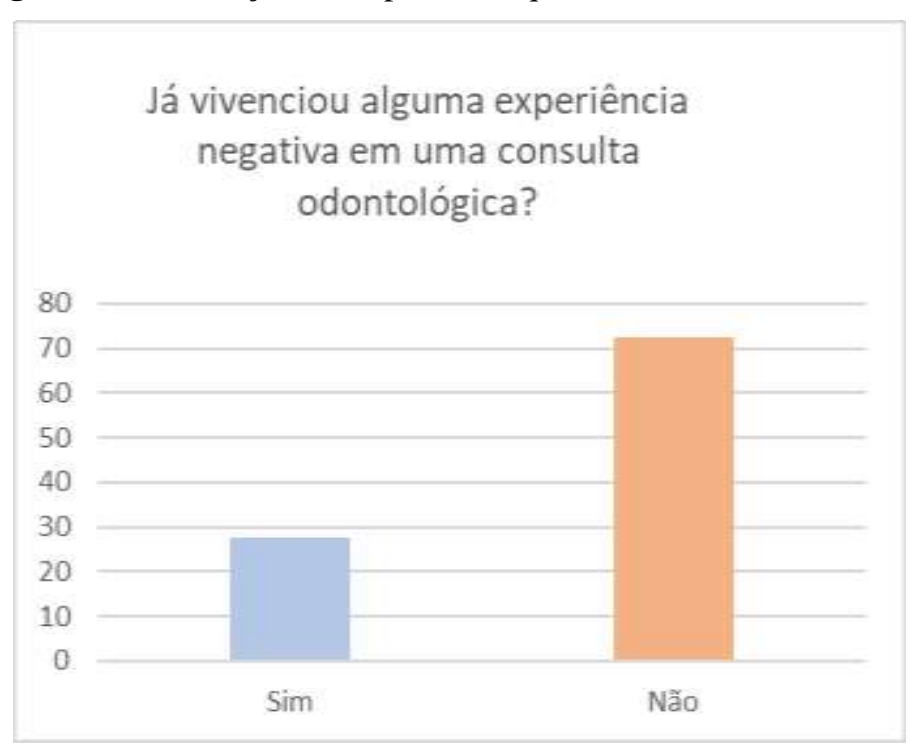

Fonte: Autores.

Foi perguntado na questão de número 4, se o participante já havia deixado de se consultar, mesmo nos casos de urgência, por medo e 13 deles (14,9\%) responderam que sim (Figura.4).

Figura 4. Distribuição de respostas da questão de número 4.

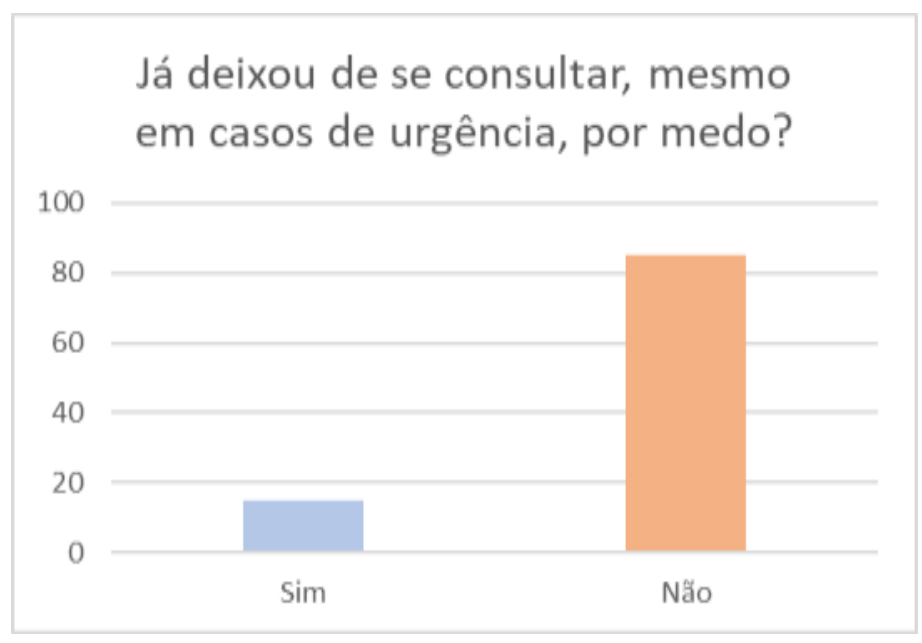

Fonte: Autores.

Na pergunta de número 5, algumas opções de atitudes e objetos de distração foram dadas aos participantes com a intenção de identificar se alguma delas lhe proporcionariam um atendimento mais tranquilo. Os participantes podiam escolher mais de uma opção. Os de maior escolha foram: conhecer o procedimento antes do mesmo ser realizado (citado 56 vezes) e ouvir música durante a consulta (citado 26 vezes) (Figura.5). 
Figura 5. Distribuição de respostas da questão de número 5.

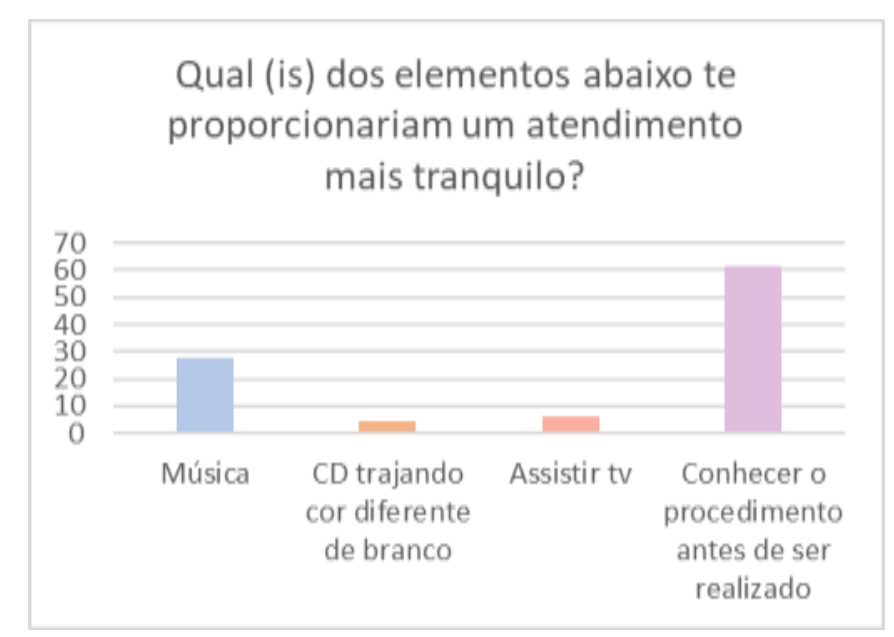

Fonte: Autores.

Sobre a espera pelo atendimento e a forma como são abordados na chegada ao consultório 34,4\% e 49,4\% dos pacientes relataram um aumento na ansiedade, respectivamente (Figura 6.).

Figura 6. Distribuição de respostas da questão de número 6.

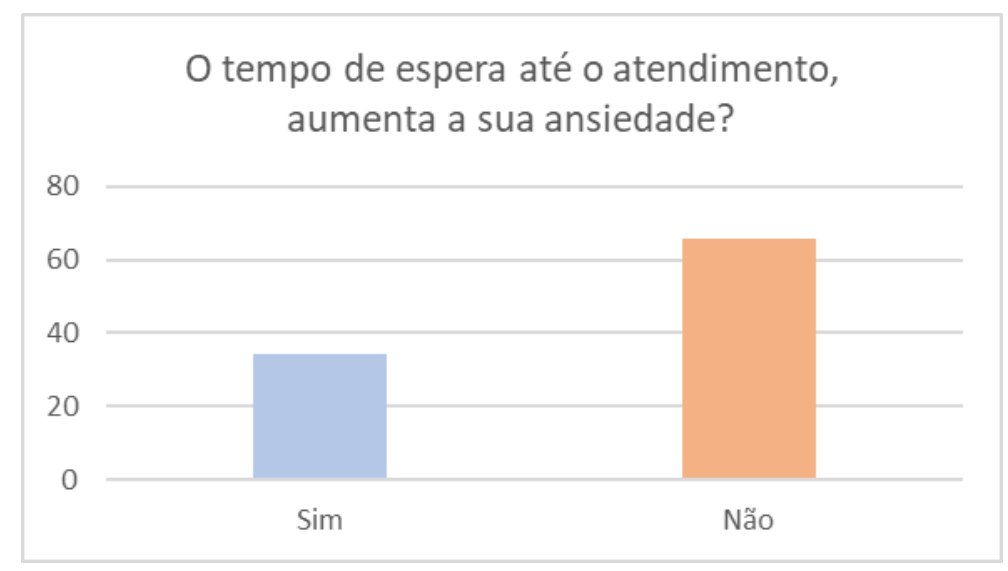

Fonte: Autores.

Total de 15 dos 87 entrevistados disseram que, quando agendam uma consulta odontológica a espera pela data agendada interfere na sua qualidade de vida (Figura 7.). 
Figura 7. Distribuição de respostas da questão de número 7

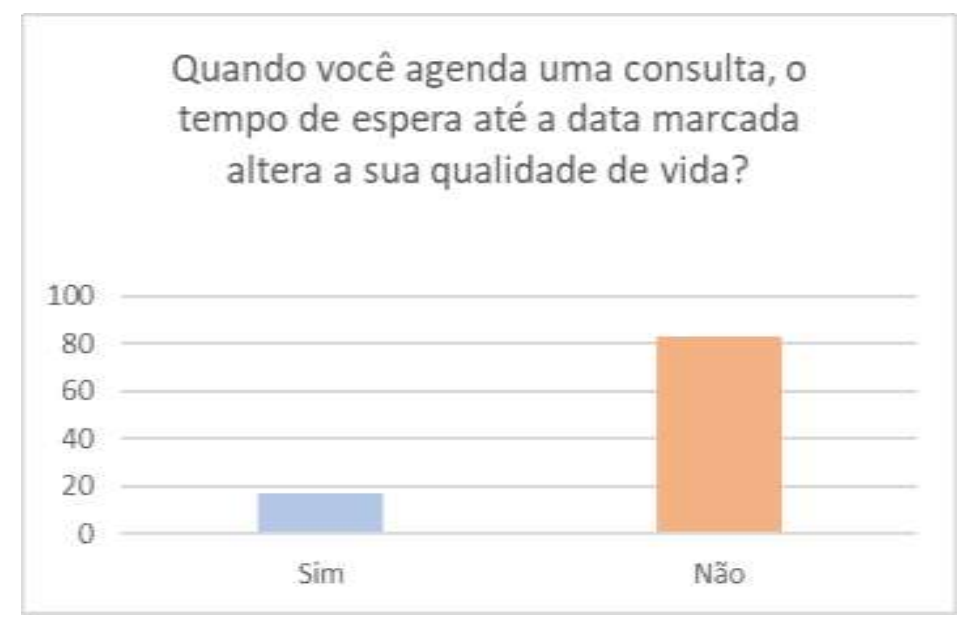

Fonte: Autores.

Foi perguntado aos participantes o quanto a postura do cirurgião dentista, desde o primeiro contato com ele, influencia no medo desenvolvido durante a consulta. 25 pessoas disseram ter muita influência e 45 pessoas alegaram não influenciar (Figura.8).

Figura 8. Distribuição de respostas da questão de número 8.

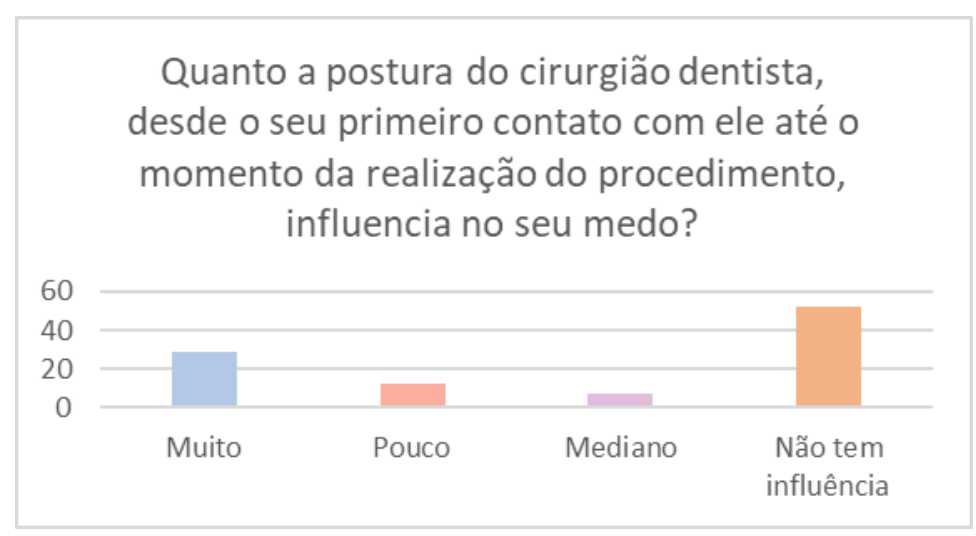

Fonte: Autores.

Na décima e última pergunta do questionário, foi abordado sobre a utilização dos equipamentos de proteção individual, principalmente durante a pandemia. 50,7\% dos participantes afirmaram se sentirem confortáveis e seguros com a utilização dos equipamentos pelos profissionais (Figura 9.). 
Figura 9. Distribuição de respostas da questão de número 9.

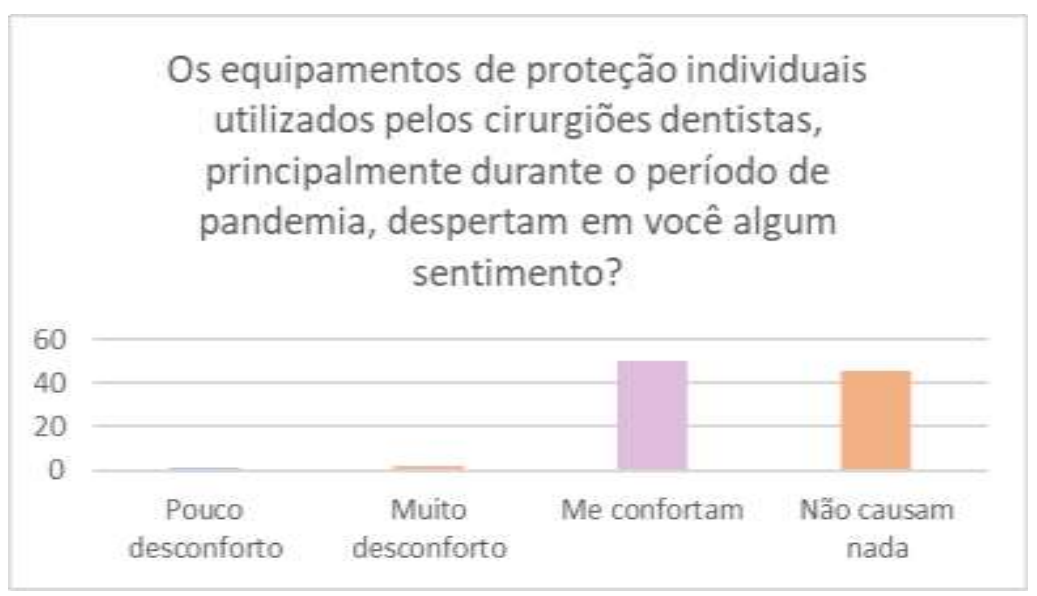

Fonte: Autores.

Mesmo com o a evolução dos tratamentos odontológicos e com o aumento da conscientização sobre a importância dos cuidados com a saúde bucal, o atendimento odontológico ainda tem sido relatado como uma condição geradora de ansiedade e estresse para muitos pacientes (Possobon et al., 2007; Possobon, Moraes, Costa Junior, \& Ambrosano, 2003), como observado nessa pesquisa, na qual 28,7\% dos pacientes apresentam medo ao atendimento odontológico. Segundo (Jeddy, Nithya, Radhika, \& Jeddy, 2018; Kanegane et al., 2003), a ansiedade odontológica é maior em mulheres, esse resultado também foi encontrado na pesquisa em questão onde 62,9\% dos entrevistados que alegaram ter medo de dentista, são mulheres. Isso pode se dar pelo fato de que, as mulheres se sentem mais confortáveis que os homens em admitir seus sentimentos.

Neste estudo a hipótese nula foi rejeitada, uma vez que, o medo de sentir dor $(32,7 \%)$ foi o fator mais apontado pelos entrevistados como responsável pelo desencadeamento de ansiedade diante do atendimento odontológico, sendo que, dos pacientes entrevistados, 27,5\% relataram já terem vivenciado alguma experiência negativa no consultório odontológico, a maioria estando relacionada a falha na técnica anestésica que lhes proporcionou um procedimento extremamente doloroso. Tais resultados estão de acordo com os achados de (de Jongh et al., 1995). Segundo de Jongh, existe uma correlação positiva entre o medo e o grau de percepção de dor em tratamentos passados e a intensidade com que estas experiências traumáticas são relatadas, ou seja, um paciente que passou por um procedimento traumático em consultas anteriores acaba desencadeando o medo e a ansiedade para os próximos atendimentos. Além disso, (Possobon et al., 2007) relatou que pacientes ansiosos tendem a superestimar a dor que sentiram e a recordar-se das experiências desagradáveis com mais intensidade do que provavelmente ocorreram, contribuindo para o resultado encontrado.

Outra questão abordada neste estudo é a esquiva ao tratamento odontológico por medo (AlQhtani \& Pani, 2019; Armfield \& Heaton, 2013). Alguns pacientes, por apresentarem o sentimento de ansiedade e medo de forma mais intensa, acabam não conseguindo frequentar o consultório odontológico para realização do tratamento necessário, como relatado por $14 * \%$ dos voluntários desta pesquisa. Como consequência de tal situação, podemos ter o agravamento do quadro do paciente, sendo necessário a realização de procedimentos mais invasivos que, consequentemente aumentam a tensão sentida pelo paciente, podendo instalar-se um ciclo vicioso de ansiedade e medo (Appukuttan, 2016; Possobon et al., 2007). Além disso, alguns paciente relatam ter maior medo quando atendidos em clínica escola por falta de experiência dos alunos (Caltabiano et al., 2018).

Em busca de alternativas que pudessem colaborar para a diminuição da ansiedade e medo frente aos tratamentos odontológicos, foi questionado aos voluntários o que seria capaz de proporcionar um atendimento mais tranquilo. Dentre as respostas, prevaleceu-se a conduta do profissional durante o atendimento, na qual 61,7\% dos voluntários responderam se 
sentirem menos ansiosos quando o profissional explica de forma clara o procedimento a ser realizado e $28,7 \%$ relataram ter muita influência a postura do cirurgião dentista na ansiedade desencadeada durante a consulta odontológica, nos permitindo observar que existe uma grande relação entre a ansiedade do paciente e a sua interação com o cirurgião-dentista (Possobon et al., 2007, 2003). (Possobon et al., 2008). Tal achado está de acordo com o estudo de (Jeddy et al., 2018), que concluiu que quanto maior o conhecimento sobre o procedimento, menor o nível de ansiedade do paciente.

Além disso, os voluntários desta pesquisa apontaram que a forma como são recebidos no consultório odontológico $(49,5 \%)$ e a espera pelo atendimento $(34,5 \%)$ podem interferir de forma negativa na ansiedade, sendo esse um tema pouco abordado em relação aos pacientes adultos, servindo como um alerta para os profissionais da área.

\section{Conclusão}

Mediante a análise dos resultados obtidos, pode-se observar que, o medo de dentista existe e os fatores responsáveis por desencadear essa insegurança começam desde o momento do agendamento da consulta. Os dados sugerem a importância de um cirurgião-dentista bem preparado tanto tecnicamente, para a realização do procedimento em si, quanto para o acolhimento do paciente em seu consultório.

\section{Agradecimentos}

Os autores negam qualquer conflito de interesse

\section{Referências}

AlQhtani, F. A., \& Pani, S. C. (2019). Parental anxiety associated with children undergoing dental treatment. European journal of paediatric dentistry: official journal of European Academy of Paediatric Dentistry, 20(4), 285-289. 10.23804/ejpd.2019.20.04.05

Appukuttan, D. P. (2016). Strategies to manage patients with dental anxiety and dental phobia: literature review. Clinical, cosmetic and investigational dentistry, 8, 35-50.10.2147/CCIDE.S63626

Armfield, J. M., \& Heaton, L. J. (2013). Management of fear and anxiety in the dental clinic: a review. Australian dental journal, 58(4), 390-407; quiz 531. doi:10.1111/adj.12118

Barasuol, J. C., Busato, C. A., Felipak, P. K., \& Menezes, J. V. N. B. (2016). Abordagem de pacientes com ansiedade ao tratamento odontológico no ambiente clínico. Revista da Associacao Paulista de Cirurgioes Dentistas.

Caltabiano, M. L., Croker, F., Page, L., Sklavos, A., Spiteri, J., Hanrahan, L., \& Choi, R. (2018). Dental anxiety in patients attending a student dental clinic. BMC oral health, 18(1), 48. 10.1186/s12903-018-0507-5

Castillo, A. R. G., Recondo, R., Asbahr, F. R., \& Manfro, G. G. (2000). Transtornos de ansiedade. Revista Brasileira de Psiquiatria, 22(suppl 2), 20-23. 10.1590/S1516-44462000000600006

De Stefano, R. (2019). Psychological factors in dental patient care: odontophobia. Medicina (Kaunas, Lithuania), 55(10). doi:10.3390/medicina55100678

de Jongh, A., Muris, P., ter Horst, G., van Zuuren, F., Schoenmakers, N., \& Makkes, P. (1995). One-session cognitive treatment of dental phobia: preparing dental phobics for treatment by restructuring negative cognitions. Behaviour Research and Therapy, 33(8), 947-954. 10.1016/0005-7967(95)00027-u

Do Nascimento, D. L., da Silva Araújo, A. C., Gusmão, E. S., \& Cimões, R. (2011). Anxiety and fear of dental treatment among users of public health services. Oral health \& preventive dentistry, 9(4), 329-337.

Doganer, Y. C., Aydogan, U., Yesil, H. U., Rohrer, J. E., Williams, M. D., \& Agerter, D. C. (2017). Does the trait anxiety affect the dental fear? Brazilian oral research, 31, e36. 10.1590/1807-3107BOR-2017.vol31.0036

Fux-Noy, A., Zohar, M., Herzog, K., Shmueli, A., Halperson, E., Moskovitz, M., \& Ram, D. (2019). The effect of the waiting room's environment on level of anxiety experienced by children prior to dental treatment: a case control study. BMC oral health, 19(1), 294. 10.1186/s12903-019-0995-y

Heyman, R. E., Slep, A. M. S., White-Ajmani, M., Bulling, L., Zickgraf, H. F., Franklin, M. E., \& Wolff, M. S. (2016). Dental fear and avoidance in treatment seekers at a large, urban dental clinic. Oral health \& preventive dentistry, 14(4), 315-320. 10.3290/j.ohpd.a36468

Jeddy, N., Nithya, S., Radhika, T., \& Jeddy, N. (2018). Dental anxiety and influencing factors: A cross-sectional questionnaire-based survey. Indian journal of dental research: official publication of Indian Society for Dental Research, 29(1), 10-15. doi:10.4103/ijdr.IJDR_33_17

Kanegane, K., Penha, S. S., Borsatti, M. A., \& Rocha, R. G. (2003). [Dental anxiety in an emergency dental service]. Revista de Saude Publica, 37(6), 786792. 10.1590/s0034-89102003000600015 
Research, Society and Development, v. 10, n.14, e142101421902, 2021

(CC BY 4.0) | ISSN 2525-3409 | DOI: http://dx.doi.org/10.33448/rsd-v10i14.21902

Lin, C.-S., Lee, C.-Y., Chen, L.-L., Wu, L.-T., Yang, S.-F., \& Wang, T.-F. (2021). Magnification of fear and intention of avoidance in non-experienced versus experienced dental treatment in adults. BMC oral health, 21(1), 328. doi:10.1186/s12903-021-01682-1

Murad, M. H., Ingle, N. A., \& Assery, M. K. (2020). Evaluating factors associated with fear and anxiety to dental treatment-A systematic review. Journal of family medicine and primary care, 9(9), 4530-4535. doi:10.4103/jfmpc.jfmpc_607_20

Pohjola, V., Rekola, A., Kunttu, K., \& Virtanen, J. I. (2016). Association between dental fear and oral health habits and treatment need among University students in Finland: a national study. BMC oral health, 16, 26. doi:10.1186/s12903-016-0179-y

Possobon, R. de F., Carrascoza, K. C., Moraes, A. B. A. de, \& Costa Jr, Á. L. (2007). O tratamento odontológico como gerador de ansiedade. Psicologia em Estudo, 12(3), 609-616. doi:10.1590/S1413-73722007000300018

Possobon, R. de F., Moraes, A. B. A. de, Costa Junior, Á. L., \& Ambrosano, G. M. B. (2003). O comportamento de crianças durante atendimento odontológico. Psicologia: Teoria e Pesquisa, 19(1), 59-64. doi:10.1590/S0102-37722003000100008

Randall, C. L., Shulman, G. P., Crout, R. J., \& McNeil, D. W. (2014). Gagging and its associations with dental care-related fear, fear of pain and beliefs about treatment. Journal of the American Dental Association (1939), 145(5), 452-458. doi:10.14219/jada.2013.50 